\title{
Single-Shot Sub-Nyquist RF Signal Reconstruction Based on Deep Learning Network
}

\author{
Shun Liu ${ }^{1,2}$, Chaitanya K. Mididoddi ${ }^{1}$, Huiyu Zhou ${ }^{3}$, Baojun $\mathrm{Li}^{2}$, Weichao $\mathrm{Xu}^{2}$, and Chao Wang ${ }^{1 *}$ \\ ${ }^{1}$ School of Engineering and Digital Arts, University of Kent, Canterbury, United Kingdom CT2 7NT \\ ${ }^{2}$ School of Automation, Guangdong University of Technology, Guangzhou, China 510006 \\ ${ }^{3}$ Department of Informatics, University of Leicester, Leicester, United Kingdom LE1 7RH \\ *Corresponding author, e-mail: c.wang@kent.ac.uk
}

\begin{abstract}
Real-time detection of high-frequency RF signals requires sophisticated hardware with large bandwidth and high sampling rates. Existing microwave photonic methods have enabled sub-Nyquist sampling for bandwidth-efficient RF signal detection but fall short in single-shot reconstruction. Here we report a novel single-shot sub-Nyquist RF signal detection method based on a trained deep neural network. In a proof-of-concept demonstration, our system successfully reconstructs high frequency multi-toned RF signals from 5x down-sampled singleshot measurements by utilizing a deep convolutional neural network. The presented approach is a powerful digital accelerator to existing hardware detectors to significantly enhance the detection capability.
\end{abstract}

Keywords- Convolutional neural network, deep learning, Nyquist sampling, single-shot, under sampling

\section{INTRODUCTION}

Processing and detection of high frequency RF signals is of crucial importance in various applications such as wireless communications, electronic warfare, large-bandwidth radar, cosmology and even ultrafast imaging. Sophisticated hardware (broadband analogue front end circuits and high-speed analogto-digital convertors) are always required to sample RF signals into digital world as per well-known Shannon-Nyquist theorem $[1,2]$. Those hardware are either expensive or suffer from limited detection bandwidth and sampling rates due to electronic bottleneck.

Microwave photonic signal processing approaches have been proposed to address this challenge by achieving subNyquist signal reconstruction. For examples, photonic time stretch (PTS) analog-to-digital conversion used chromatic dispersion to slow down fast RF signals carried with pre-chirped optical pulses [3]. Built on this concept, an anamorphic stretch approach can further reduce the signal time-bandwidth product (TBWP) by selectively stretching the high-frequency features further $[4,5]$. One of the major challenges for stretch-type methods is that they only work for time-gated RF signals to allow them to be further stretched. Recently, photonic compressive sensing has shown great potential in bandwidthcompressed RF signal detection [6-8]. However, compressive sensing approach suffers from the following fundamental problems: (1) high-speed random bit sequences are always required; (2) it only works for spectrum-sparse RF signals; (3) it

This work was supported in part by the EU FP7 Marie-Curie Career Integration Grant (631883), in part by the Royal Society (IE170007), in part by National Natural Science Foundation of China (Projects 61771148 ,

61571211 and U1501251), and in part by Guangzhou Science and Technology Plan (Project 201607010290) falls short in single-shot real-time signal detection as it requires the same signal to be mixed with various pre-set random sequences for the reconstruction purpose, which is not always feasible in many practical scenarios such as frequency hopping spread spectrum systems. Recent research efforts on phaseshifted Fourier domain scanning [9] have been made to address the first two issues. However, the third one remains an unsolved challenge. Therefore, new methods to achieve significant subNyquist sampling for single-shot high-frequency RF signal detection are highly desirable.

Recently, deep learning [10], which is a new class of machine learning methods using deep layered neural networks for automated data analysis, has attracted wide interests and found rich applications in signal processing [11], spectrum measurement [12], microscopy [13] and object classification [14]. Here we propose and demonstrate the first use of a deep convolutional neural network in sub-Nyquist temporal signal reconstruction, significantly enhancing its detection bandwidth for high-frequency RF signals. In our trained network, the only input is under-sampled serial data captured in a single-shot using a normal low-speed real-time oscilloscope, without any extra signal mixing as used in the existing microwave photonic solutions [6-9]. Therefore, single-shot sub-Nyquist RF signal reconstruction with significant data compression or bandwidth enhancement can be achieved. In a proof-of-concept experiment, we blindly tested the trained deep learning model using various unknown RF signals with randomly selected multitoned carrier frequencies. Satisfactory reconstruction of a 5-tone RF signal $(0.86875 \mathrm{GHz}, 0.9375 \mathrm{GHz}, 1.0375 \mathrm{GHz}, 1.10625 \mathrm{GHz}$, and $1.13125 \mathrm{GHz}$ ) has been achieved from 1.8x under sampling at $1.25 \mathrm{GS} / \mathrm{s}$. The presented approach functions as a powerful digital accelerator to the existing hardware detectors to enhance the detection capability. Our method can be applicable to many other fields beyond microwave photonics, where high-speed temporal signal detection is essential.

\section{DeEP LEARNING Model ARCHITECTURE}

Details beyond the Nyquist frequency of the under-sampled signals are unable to be reconstructed if we utilize traditional methodologies, e.g. Whittaker-Shannon interpolation or spline interpolation. In this section, we propose a very deep convolutional neural network inspired by Kim et.al. [15]. The configuration of our network model, which consists of three 


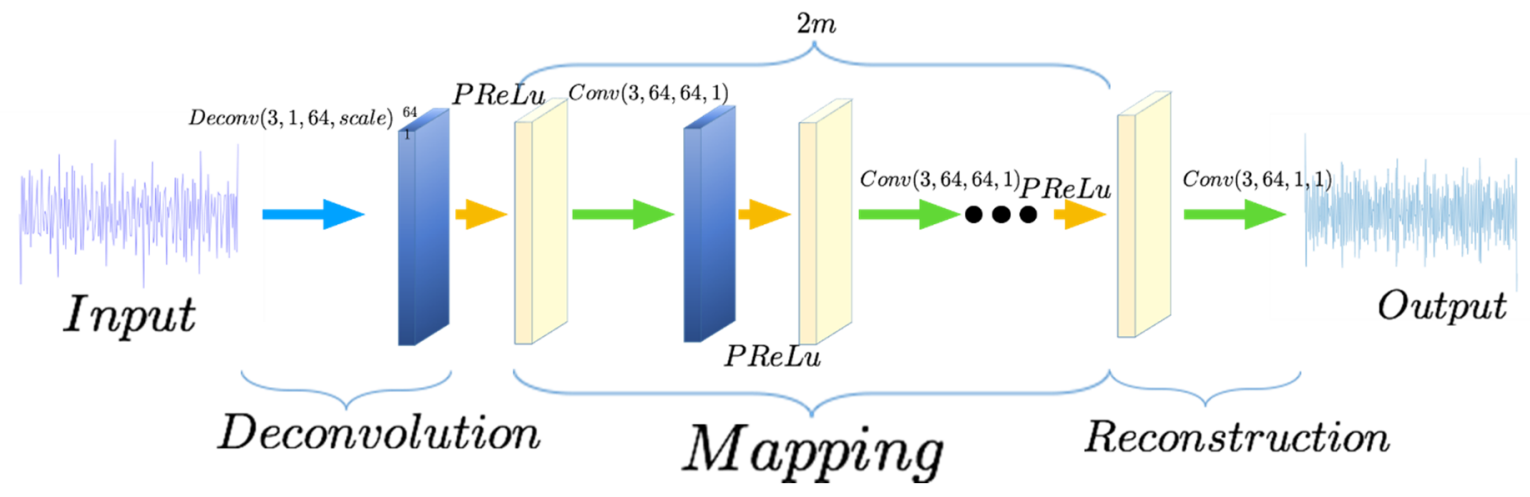

Figure 1 : Structure of our deep neural network. Given an under-sampled input data stream, the first layer, namely Deconvolution, samples them up to a series of feature maps. It is worth noting that the deconvolutional stride of Deconvolution is equal to the scale, which depends on the magnification factor, e.g., 2X, 3X of our model. After a non-linear mapping layer PReLU, we stack $m$ pairs of layers (convolution and nonlinear) with the same structure consecutively to construct the second part. The last layer combines predictions within different channels to produce the final signal with relatively perfect integrity.

main parts: Deconvolution, Mapping and Reconstruction, is outlined in Fig. 1. The first part is Deconvolution (also known as convolutional transpose) layer, which projects low-dimensional data (Input) to 64 feature maps with the same size as the reconstructed Output. This scaling up process prepares the input data for subsequent mapping and reconstruction steps. The last layer of the reconstruction step is just a general convolutional layer. In the Mapping step, in order to simplify our model, we cascade $m$ convolutional layers with each being followed by an individual PReLU layer [16]. For better understanding, we separately denote convolutional layers as $\operatorname{Conv}\left(f_{i} ; c_{i} ; n_{i} ; s_{i}\right)$, and deconvolutional layers as $\operatorname{Deconv}\left(f_{i} ; c_{i} ; n_{i} ; s_{i}\right)$, where the variables $f_{i} ; c_{i} ; n_{i} ; s_{i}$ represent the filter size, the number of channels, the number of filters and stride in the $i^{\text {th }}$ layer, respectively. To properly carry out convolutional operation, neighbouring points need to keep the same size for all the features maps (including the output signals) [17]. This is done by filling up the maps with zeros for missing elements.

\section{DATA PREPARATION AND TRAINING Details}

Since all periodic 1D signals can be modelled as superposition of multiple sinusoidal waves with different phases and amplitudes, in this work we decide to use superposition of sinusoidal signals with various frequency components and amplitudes to generate the training set for our network. We aim to reconstruct a section of multitoned RF signal with total 1000 sampled points from an under-sampled Input data with only 200 sampled points. Let $\mathcal{A} \in[0(0.1) 1]$ and $\mathcal{F} \in[100(1) 200]$ denote the normalized amplitudes and frequency values of each sine waves respectively. In addition, the number of frequency components in each sinusoidal signal, denoted by $\mathcal{N} \epsilon$ [5(1)30], is also randomly selected to produce the whole training set. The above notation $\left[h_{1}(\Delta h) h_{2}\right]$ stands for a list of $h$ starting from $h_{1}$ to $h_{2}$ with the increment of $\Delta h$. Then each sample $X$ in our training set can be generated as follows:

$$
X=\sum_{i=1}^{\mathcal{N}} \mathcal{A}_{i} \sin \left(2 \pi \mathcal{F}_{i} t\right)
$$

Equation (1) indicates that our training samples are produced from the combination of multiple randomly selected sinusoidal signals, which are characterized by three variables $\mathcal{A}, \mathcal{F}$ and $\mathcal{N}$. This leads to good diversity of our training data set and better inclusivity of our model. With Eq. (1) we generate a total of $8 \times$ $10^{5}$ training samples for our deep neural network model. Each sample involves a 200-point under-sampled signal as the Input to the model and a 1000-point Nyquist sampled signal as the Target to guide the training. Note that $\left\{\mathcal{F}_{1}, \ldots \mathcal{F}_{\mathcal{N}}\right\}$ is a selected set of frequency values to produce one training sample. According to the Nyquist-Shannon sampling theorem, at least $2 \times \max \left\{\mathcal{F}_{1}, \ldots \mathcal{F}_{\mathcal{N}}\right\}$ points are required if one would like to restore the original signal without losing any frequency components. Therefore, the produced training data sets well represent sub-Nyquist sampling. Note that although we use a

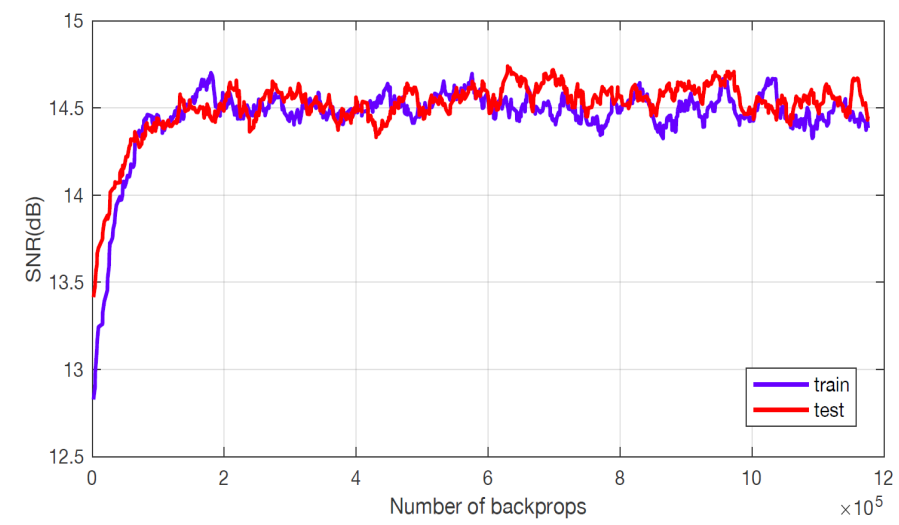

Fig. 2. Performance of our trained deep convolutional neural network. An average SNR of $14.5 \mathrm{~dB}$ has been achieved in both our training and testing processes.

fixed signal length in the training process, the deep convolutional neural network presented here can be further applied on signals of arbitrary length during testing.

Now we describe the objective function to be minimized in an attempt to find optimal parameters of our model shown in Fig. 1. Let $x$ and $y$ denote an under-sampled signal and an 'original signal' respectively. Given a training set $\left\{x^{(i)}, y^{(i)}\right\}_{i=1}^{N}$, our goal 

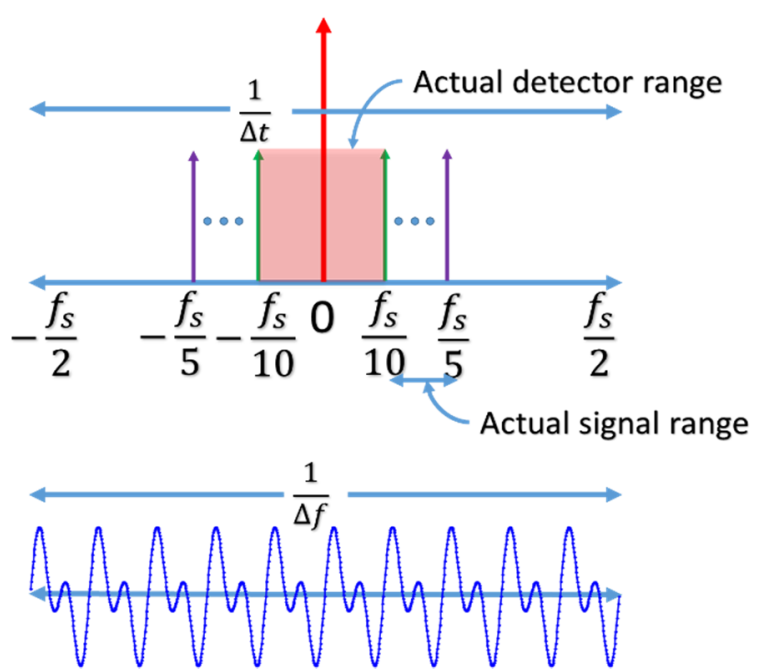

Fig. 3. Frequency model. The figure explains the input range tested for deep learning model. Actual detector range: The maximum frequency range that can be detected by under sampled detector. $\Delta t=$ sampling time between adjacent points in temporal domain of the actual high frequency signal under nyquist theorem. $f_{s}=\frac{1}{\Delta t}$ is the sampling frequency, width of entire frequency window. $\frac{1}{\Delta f}=$ Time period of the signal.

is to learn a model $f$ that predicts values $\hat{y}=f(x, \theta)$, where $\hat{y}$ is an estimated signal and $\theta$ is the set of parameters (including weights and bias) in our model. The model function $f$ is attained by optimizing a classical loss function - mean squared error (MSE) between the estimated and the original signals averaged over the training set, as shown in the following equation.

$$
\text { Loss }=\frac{1}{N} \sum_{i=1}^{N}\left(y^{(i)}-f\left(x^{(i)}, \theta\right)\right)^{2}
$$

We used a network with total number of layers being $m=$ 18. Training uses batches of size 64 and Adam algorithm [18] for optimization. We set the initialized learning rate to $10^{-4}$. Then training is regularized by the weight decay ( $L 2$ penalty multiplied by $10^{-4}$ ). For the weight initialization, we use the method developed by He et al [16]. We train all the experiments over 100 epochs, which takes roughly 4 days on a GPU Tesla K80 platform. We implement our model using a TensorFlowGPU package [19].

Performance of our trained network has been evaluated using both training and testing data in terms of Signal-to-Noise Ratio (SNR) of reconstructed signals. $95 \%$ of the produced data are used to train the model and the rest $5 \%$ are used to test our model at the same time. Performance evaluation results are shown in Fig. 2. These two convergence curves in the figure explicitly point out that the designed model is effective and without overfitting.

\section{EXPERIMENT OF DEEP LEARNING RF SigNAL DETECTION}

A proof-of-the-concept experiment has been carried out to verify the utility of our trained deep learning network in the single-shot sub-Nyquist RF signal reconstruction. In the experiment, unknown analogue RF signals with randomly selected multi-tones are generated using an arbitrary waveform generator (Tektronix AWG7122C). RF signals are back-to-back measured using a real time digital oscilloscope (Tektronix
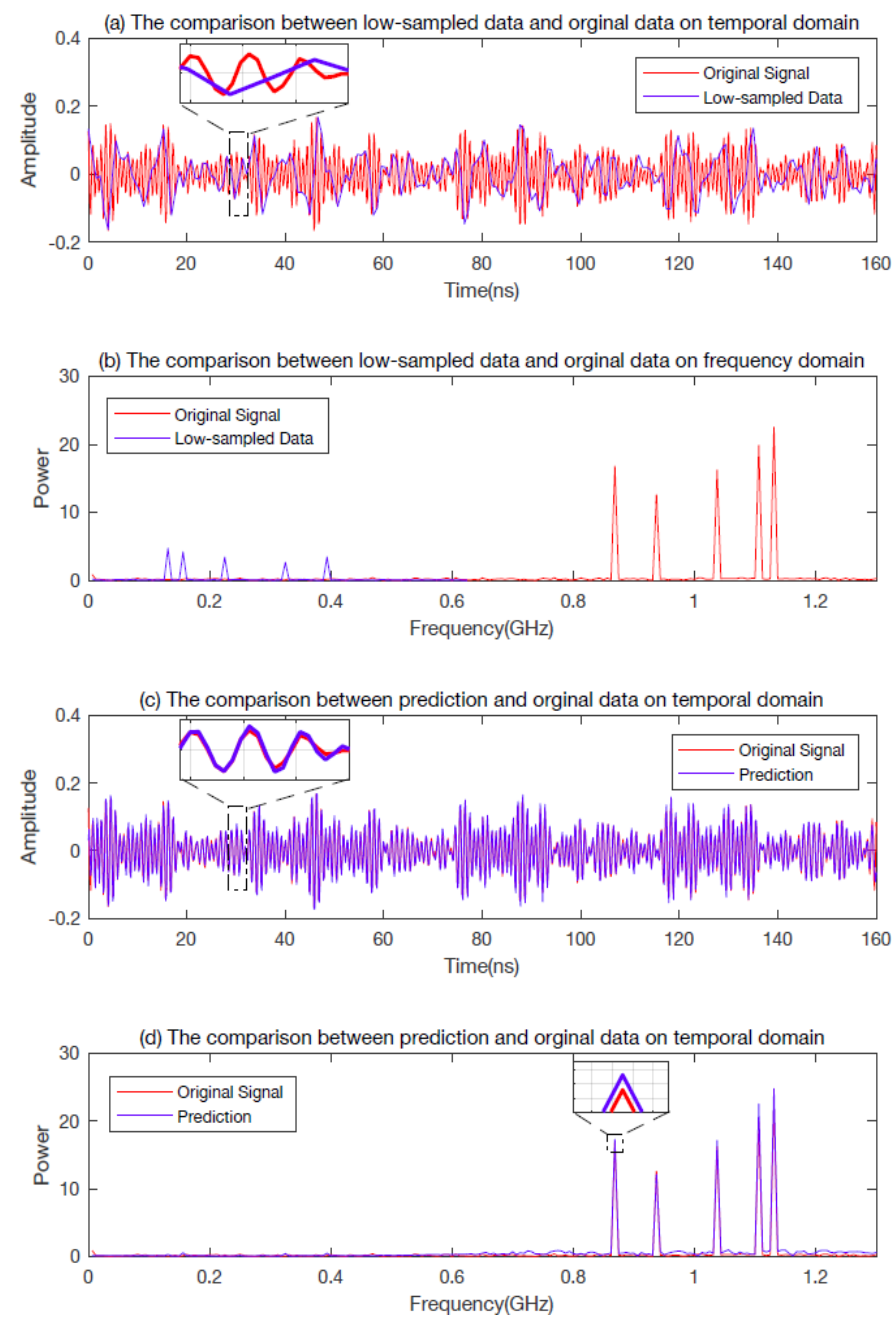

Fig. 4. Top two is the low-sampled data plotted on temporal and frequency domain compares with the original data, it is obvious that most of the highfrequency details are missing due to the insufficient sampling rate. In contrast, the bottom two show that the signal reconstructed by our model is very similar to the original signal.

DPO72304DX) under two different detection settings to obtain the original and under-sampled signals.

In this section, we evaluate the performance of our model on signals collected from oscilloscope in terms of Signal-Noise Ratio (SNR). A 5-tone RF signal with carrier frequencies of $(0.86875 \mathrm{GHz}, 0.93750 \mathrm{GHz}, 1.03750 \mathrm{GHz}, 1.10625 \mathrm{GHz}$, and $1.13125 \mathrm{GHz}$ ) has been considered to exemplify our model. The frequency model is described in Fig. 2. Considering that the actual sampling rate $\left(f_{s}\right)$ of the AWG is set as $6.25 \mathrm{GS} / \mathrm{s}$, the tones are considered to be within the discrete range $\left[\frac{f_{s}}{10}(\Delta f) \frac{f_{s}}{5}\right]$ to satisfy the model's normalized frequency criterion [100(1)200] along the total 1000-point spectrum, where $\Delta f$ is the frequency resolution $(6.25 \mathrm{MHz}$ in our case). Based on this frequency model, the multiple RF tones are located at 139,150, 166,177, and 181 on the normalized frequency range of $-499: 500$. The length of individual target signal is considered to be 1000 points with $160 \mathrm{ps}$ as the sampling time in the real-time oscilloscope (Setting one). To produce the testing signal, the same 
oscilloscope captures the same multi-tone RF signal again with a $5 x$ down-sampling rate of $1.25 \mathrm{GS} / \mathrm{s}$ (Setting two), leading to the actual under-sampled signal with a length of only 200 points. Note that the under-sampled testing signal is captured in a singleshot measurement and is the only input to our trained deep neural network to reconstruct the original signal. Therefore single-shot sub-Nyquist RF signal reconstruction can be achieved in our approach.

Reconstruction results are shown in Fig. 4. Figure 4(a) compares the original RF signal (1000 points) to the directly down-sampled signal (200 points). It is clearly evident that down-sampling has lost many high-frequency components, which can be confirmed by the frequency domain results as shown in Fig. 4(b), where only fake low-frequency components can be restored. On the other hand, with our trained deep neural network, the original signal has been faithfully reconstructed from under-sampled single-shot measurement with SNR of 14 dB, as confirmed by Figs. 4(c) and (d).

\section{CONCLUSION}

We have proposed and experimentally demonstrated a novel approach for single-shot sub-Nyquist reconstruction of high frequency RF signals with $5 x$ down-sampled detector. This was made possible based on the first use of deep convolutional neural network in super-pixel 1D signal reconstruction. To train our deep learning model, a big sample set has been produced based on random frequency combination. With the trained model, a multi-tone RF signal has been faithfully reconstructed from $5 \mathrm{x}$ down-sampled single-shot measurement. This approach can significantly enhance the detection capability of existing hardware detectors and hence holds great promise in many fields of microwave photonics, where high-speed temporal signal detection is essential.

\section{REFERENCES}

[1] R. H. Walden, "Analog-to-digital converter survey and analysis," IEEE J. Sel. Areas Commun., vol 17, pp. 539-550, Apr. 1999.

[2] G. C. Valley, "Photonic analog-to-digital converters," Opt. Express, vol. 15, no. 5, pp. 1955-1982, Mar. 2007.

[3] Y. Han and B. Jalali, "Photonic time-stretched analog-to-digital converter: Fundamental concepts and practical considerations," J. Lightwave Technol., vol. 21, no. 12, pp. 3085-3103, Dec. 2003.
[4] B. Jalali, J. Chan, and M. H. Asghari, "Time-bandwidth engineering," Optica, vol. 1, no. 1, pp 23-31, Jul. 2014.

[5] C. K. Mididoddi and C. Wang, "Adaptive non-uniform photonic time stretch for blind RF signal detection with compressed time-bandwidth product," Opt. Commun., vol 396, pp 221-227, Aug. 2017.

[6] B. T. Bosworth and M. A. Foster, "High-speed ultrawideband photonically enabled compressed sensing of sparse radio frequency signals," Opt. Lett., vol. 38, no. 22, pp. 4892-4895, Nov. 2013.

[7] H. Chi, Y. Chen, Y. Mei, X. Jin, S. Zheng, and X. Zhang, "Microwave spectrum sensing based on photonic time stretch and compressive sampling," Opt. Lett., vol. 38, no. 2, pp. 136-138, Jan. 2013.

[8] C. K. Mididoddi, F. Bai, G. Wang, J. Liu, S. Gibson, and C. Wang, "Highthroughput photonic time-stretch optical coherence tomography with data compression," IEEE Photonics J., vol. 9, no. 4, pp. 1-15, Aug. 2017.

[9] Q. Guo, H. Chen, Y. Wang, Y. Guo, P. Liu, X. Zhu, Z. Cheng, Z. Yu, S. Yang, M. Chen, and S. Xie, "High-Speed Compressive Microscopy of Flowing Cells Using Sinusoidal Illumination Patterns," IEEE Photonics J, vol. 9, no. 1, pp. 1-11, Feb. 2017.

[10] Y. LeCun, Y. Bengio, and G. Hinton, "Deep learning," Nature, vol. 521, no. 7553, pp 436, May 2015.

[11] D. Yu and L. Deng, "Deep Learning and Its Applications to Signal and Information Processing [Exploratory DSP]," in IEEE Signal Processing Magazine, vol. 28, no. 1, pp. 145-154, Jan. 2011

[12] J. Liu, M. Osadchy, L. Ashton, M. Foster, C. J. Solomon and S. J. Gibson, "Deep convolutional neural networks for Raman spectrum recognition: a unified solution," Analyst, vol. 142, pp. 4067-4074, 2017.

[13] Y. Rivenson, Z. Grcs, H. Gnaydin, Y. Zhang, H. Wang, and A. Ozcan, "Deep learning microscopy," Optica, vol. 4, no. 11, pp 1437-1443, Nov. 2017.

[14] C. L. Chen, A. Mahjoubfar, L.-C. Tai, I. K. Blaby, A. Huang, K. R. Niazi, and B. Jalali, "Deep Learning in Label-free Cell Classification," Sci. Rep., vol. 6, no. 21471, Mar. 2016.

[15] J. Kim, J. Kwon Lee, and K. Mu Lee, "Accurate image super-resolution using very deep convolutional networks," in Proceedings of the IEEE Conference on Computer Vision and Pattern Recognition, pp. 1646-1654, Jun. 2016.

[16] K. He, X. Zhang, S. Ren, and J. Sun, "Delving deep into rectifiers: Surpassing human-level performance on imagenet classification," in Proceedings of the IEEE international conference on computer vision, pp. 1026-1034, Dec. 2015.

[17] K. He, X. Zhang, S. Ren, and J. Sun, "Deep residual learning for image recognition," in Proceedings of the IEEE conference on computer vision and pattern recognition. pp. 770-778, June 2016.

[18] D. P. Kingma and J. Ba, "Adam: A method for stochastic optimization," arXiv preprint arXiv:1412.6980, 2014

[19] M. Abadi, P. Barham, J. Chen, Z. Chen, A. Davis, J. Dean, M. Devin, S. Ghemawat, G. Irving, M. Isard et al., "Tensorflow: A system for largescale machine learning." In OSDI, vol. 16, pp. 265-283. 2016. 